\title{
Fracture of disordered solids in compression as a critical phenomenon. II. Model Hamiltonian for a population of interacting cracks
}

\author{
Renaud Toussaint* and Steven R. Pride ${ }^{\dagger}$ \\ Géosciences Rennes, Université de Rennes 1, 35042 Rennes Cedex, France \\ (Received 14 November 2001; revised manuscript received 13 June 2002; published 27 September 2002)

\begin{abstract}
To obtain the probability distribution of two-dimensional crack patterns in mesoscopic regions of a disordered solid, the formalism of Paper I requires that a functional form associating the crack patterns (or states) to their formation energy be developed. The crack states are here defined by an order parameter field representing both the presence and orientation of cracks at each site on a discrete square network. The associated Hamiltonian represents the total work required to lead an uncracked mesovolume into that state as averaged over the initial quenched disorder. The effect of cracks is to create mesovolumes having internal heterogeneity in their elastic moduli. To model the Hamiltonian, the effective elastic moduli corresponding to a given crack distribution are determined that includes crack-to-crack interactions. The interaction terms are entirely responsible for the localization transition analyzed in Paper III. The crack-opening energies are related to these effective moduli via Griffith's criterion as established in Paper I.
\end{abstract}

DOI: $10.1103 /$ PhysRevE.66.036136

PACS number(s): 62.20.Mk, 46.50.+a, 46.65.+g, 64.60.Fr

\section{INTRODUCTION}

In triaxial-stress experiments on rocks in the brittle regime, the onset of a macroscopic localization of deformation is usually observed around peak stress [c.f. Bésuelle [1] for a review]. Such departure from a macroscopically uniform deformation regime is intrinsically beyond the capacities of a mean-field theory, and so a specific model is developed here that takes the orientational nature of crack-to-crack interactions into account.

This is the second paper in a series of three dedicated to exploring how the physical properties of disordered solids evolve as they are led to failure in a state of compression. The goal of this paper is to obtain a reasonable form for the Hamiltonian $E_{j}\left(\boldsymbol{\varepsilon}, \boldsymbol{\varepsilon}_{m}\right)$ which is defined as the average work required to lead an intact region at zero deformation to the crack state denoted by $j$ when the maximum applied strain is $\boldsymbol{\varepsilon}_{m}$ and where the final strain $\boldsymbol{\varepsilon}$ is possibly different than $\boldsymbol{\varepsilon}_{m}$ due to a final unloading. This Hamiltonian must be expressed in terms of the spatial distribution of the local order parameter that is the variable used to characterize the population of cracks in each mesovolume of a huge disordered-solid system.

Most existing lattice models explore the dynamics of scalar order parameters either representing the breakdown of elastic spring or beam networks under tensile stress [2], or of fuse networks [3]. The analogies between such scalar models and fracture of disordered media has been widely discussed [4]. One advantage of our approach is the ability to explore interactions based on a fully tensorial description of the stress perturbations produced by each crack. Another is its ability to yield analytical rather than only numerical results.

\footnotetext{
*Present address: Department of Physics, University of Oslo, P.O. Box 1048 Blindern, 0316 Oslo 3, Norway. Email address: Renaud.Toussaint@fys.uio.no

${ }^{\dagger}$ Email address: Steve.Pride@univ-rennes1.fr
}

Using $E_{j}$ in the partition function established in Paper I, it is possible to explore the crack patterns that emerge in compressive settings for which isolated cracks appear in an intrinsically stable manner no matter their size [5], and for which macroscopic localization is a collective phenomenon due to the energetic organization of small cracks as opposed to an instability associated with the largest defects. In the present paper, we retain the leading-order effects of orientedcrack populations interacting in two-dimension (2D). The overriding importance of the long-range elastic interactions leaves hope that 3D generalizations would not yield qualitatively different critical behavior.

\section{PRINCIPLES OF THE MODEL}

\section{A. Order-parameter definition}

We now elaborate on the crack model introduced in Paper I. Each mesovolume of a huge rock system is discretized into a square network of diamond-shaped cells of size $\Lambda$ (grain sizes) and only a single crack is potentially present in each cell. A crack is located at the center of the cell and has a length $d$ somewhere within the support $\left[0, d_{m}\right]$, where $d_{m}$ is the maximum crack length (a fixed parameter of the system) required to satisfy $d_{m}<\Lambda$. In the perturbative treatment of the crack interactions developed herein, $\epsilon=\left(d_{m} / \Lambda\right)^{D}$ is taken to be a small number, where $D$ is the number of space dimensions (in the present model, $D=2$ ). The local order parameter $\varphi(\boldsymbol{x})$ associated with each cell $\boldsymbol{x}$ is taken to have an amplitude $\psi=|\varphi|=\left(d / d_{m}\right)^{D}$ and has a sign that indicates whether the crack is oriented at $+45^{\circ}$ or $-45^{\circ}$ relative to the principal stress direction (the so-called "axial direction"). The model is summarized in Fig. 1

The restriction that cracks are either at $\pm 45^{\circ}$ and have lengths less than the grain dimensions is of course a great simplification compared to what is found inside of real rocks. However, we only need to characterize the essential features of a crack population that contribute to localization phenomena and, to this extent, it appears overly complicated to 


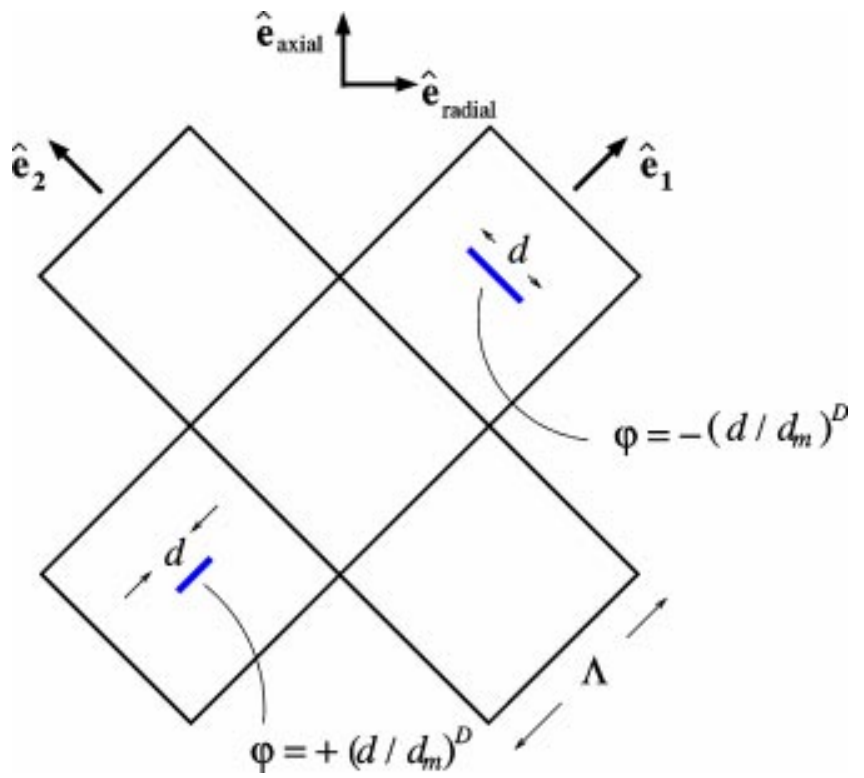

FIG. 1. Part of the diamond network of cells that comprise a mesovolume. Each cell has the linear dimension $\Lambda$ and is only allowed to contain one crack. The maximum length of any crack is $d_{m}$ and this length is assumed to be sufficiently small that $\left(d_{m} / \Lambda\right)^{D} \ll 1$. The amplitude of the order parameter is by definition $\psi=|\varphi|=\left(d / d_{m}\right)^{D}$, where $d$ is the length of the crack found in the cell, while the sign of $\varphi$ indicates the orientation of the crack as shown.

model the amazing variety of crack geometries encountered in real rocks $[6,7]$. The localization transition involves spontaneous breaking of symmetries both under translation and parity (inversion of the minor stress axis) as is seen from the structure of the shear bands emerging in the post-peak-stress regime [8]. The essential feature of any proposed order parameter is that it must reflect and quantify the amount of local symmetry breaking and our simple model with cracks at either $\pm 45^{\circ}$ does just this.

Furthermore, there is evidence both from acousticemissions monitoring [9] and from direct observation after unloading [10], that cracks developing prior to peak stress do not exceed an extent of a few grains diameters. This is principally because the grain contacts that break are much weaker than the grains and have a finite length so that cracks arriving in compression do so stably [5]. Crack coalescence is not explicitly allowed for. However, since several neighboring cells in a line may all contain cracks of the same sign, the long-range elastic effect of long (coalesced) cracks is effectively allowed for. Our picture of the final shear bands experimentally observed in the post-peak-stress regime is that they were created by unstable sliding along a band weakened in the pre-peak-stress regime by a concentration of coherently oriented cracks [8]. Our model allows small cracks to stably concentrate en échelon along conjugate bands relative to the principal-stress direction; however, it does not model the final unstable rupture along a given band.

\section{B. Formation energy of a crack pattern}

It has been established in Paper I that to a reasonable approximation, the work required to form a crack state, as averaged over the initial disorder, separates into one part representing the work required to break the grain contacts, and a second part representing the elastic energy stored in the cracked solid. This was expressed in Eqs. (26)-(28) of Paper I as

$$
E_{j}=\frac{q}{2} \boldsymbol{\varepsilon}_{m}:\left(\mathbf{C}_{0}-\mathbf{C}_{j}\right): \boldsymbol{\varepsilon}_{m}+\frac{1}{2} \boldsymbol{\varepsilon}: \mathbf{C}_{j}: \boldsymbol{\varepsilon}
$$

The first term of Eq. (1) is the energy spent in the irreversible formation of the crack state $j$ averaged over quenched disorder and was obtained through an application of Griffith's principle. The parameter $q$ derives from the quencheddisorder distribution and lies in the range $[1 / 2,1]$ (see Sec. III B 2 of Paper I). The second term is the reversibly-stored elastic energy with $\mathbf{C}_{j}$ being the elastic-stiffness tensor of state $j$.

Our principal task is therefore to model the way that cracks and collective crack-states affect the overall elastic moduli of a mesovolume. This requires detailed knowledge of the stress (or strain) field throughout the mesovolume in the presence of arbitrary crack populations, and we treat this need using the following approximations. First, since the cracks in the model are isolated one to each cell, their main effect regarding the far-field stress is to change the elastic moduli of their embedding cell. Such a change is modeled assuming the cracks to be penny-shaped ellipsoidal cavities. We ignore how such ellipses change shape when the applied stress is unloaded/reloaded since linear elasticity alone captures the principle effect of how the rock becomes weaker due to strategic placement of cracks in cells. Since a crack occupies a limited extent of a cell, the modification of the moduli is small compared to the moduli of the intact cell so that the resulting far-field stress field can be developed as a Born series. It is in the third term of this development that crack-to-crack interactions are first allowed for. Higher-order interactions (three cracks simultaneously interacting and so on) are negligible to the extent that $\epsilon=\left(d_{m} / \Lambda\right)^{D}$ can be considered small.

\section{ELASTIC ENERGY}

The goal of this section is to determine the elastic energy $E_{j}^{\mathrm{el}}$ stored in a mesovolume occuping the region $\Omega$ and containing the crack state $j$ (which denotes the spatial distribution of $\varphi(x)$ at all points $\boldsymbol{x}$ of $\Omega$ ) when a displacement corresponding to a uniform strain tensor $\boldsymbol{\varepsilon}^{(0)}$ is applied on the external surface $\partial \Omega$ of the mesovolume.

\section{A. Elastic energy of a weakly heterogeneous solid}

The effect of the crack field $\varphi(x)$ is to perturb the stiffness tensor of each cell as $\mathbf{C}(\boldsymbol{x})=\mathbf{C}^{0}+\delta \mathbf{c}[\varphi(\boldsymbol{x})]$, where $\mathbf{C}^{0}$ denotes the moduli of an uncracked cell (assumed uniform for all cells), and where $\delta \mathbf{c}(\boldsymbol{x})$ is a small perturbation due to the possible presence of a crack as characterized by $\varphi(x)$. It is established in the Appendix that the nonzero components of $\delta \mathbf{c}$ are typically smaller than those of $\mathbf{C}^{0}$ by a factor $\epsilon$ $=\left(d_{m} / \Lambda\right)^{D} \ll 1$. Our problem is to resolve an elasticity 
boundary-value problem in a region $\Omega$ containing a weakly heterogeneous stiffness tensor $\mathbf{C}(\boldsymbol{x})$.

The displacement boundary conditions are given as

$$
\forall x \in \partial \Omega, \quad \mathbf{u}(x)=\varepsilon^{(0)} \cdot x,
$$

where $\boldsymbol{x}$ denotes distance from the center of the mesovolume. Elastostatic equilibrium requires that

$$
\partial_{j} \tau_{i j}=C_{i j k l}^{0} \partial_{j} \partial_{k} u_{l}+\partial_{j}\left[\delta c_{i j k l} \partial_{k} u_{l}\right]=0
$$

throughout $\Omega$, where summation over repeated indices is assumed both here and throughout. Due to the linearity of the problem, we use the elastostatic Green tensor $\mathbf{G}=G_{i j} \hat{\boldsymbol{x}}_{i} \hat{\boldsymbol{x}}_{j}$ for a uniform material which is a solution of

$$
\begin{gathered}
C_{i j k l}^{0} \partial_{j} \partial_{k} G_{l m}\left(\boldsymbol{x}, \boldsymbol{x}^{\prime}\right)+\delta_{i m} \delta^{D}\left(\boldsymbol{x}-\boldsymbol{x}^{\prime}\right)=0, \\
\forall \boldsymbol{x} \in \partial \Omega, \quad G_{i j}\left(\boldsymbol{x}, \boldsymbol{x}^{\prime}\right)=0 .
\end{gathered}
$$

The components $G_{i j}\left(\boldsymbol{x}, \boldsymbol{x}^{\prime}\right)$ define the $i$ th component of the displacement at $\boldsymbol{x}$ induced by a unit point force acting along the $j$ axis at $\boldsymbol{x}^{\prime}$. Here, $\delta_{i j}$ is the Kronecker symbol, and $\delta^{D}$ is the $D$-dimensional Dirac distribution.

The solution for the displacements when no cracks are present is simply $\mathbf{u}^{(0)}(\boldsymbol{x})=\boldsymbol{\varepsilon}^{(0)} \cdot \boldsymbol{x}$ throughout all of $\Omega$. Thus, it is a straightforward excercise to demonstrate that the total displacement $\mathbf{u}$ in the presence of the cracks satisfies the following integral equation:

$$
u_{i}(\boldsymbol{x})=u_{i}^{(0)}(\boldsymbol{x})+\int_{\Omega} G_{i j}\left(\boldsymbol{x}, \boldsymbol{x}^{\prime}\right) \partial_{k^{\prime}}\left[\delta c_{j k l m} \partial_{l^{\prime}} u_{m}\right]\left(\boldsymbol{x}^{\prime}\right) d^{D} \boldsymbol{x}^{\prime},
$$

where $\partial_{i}$, denotes the partial derivative relative to the coordinate $x_{i}^{\prime}$. Using $\epsilon$ as the argument of a series expansion, we write the displacements as $\mathbf{u}=\mathbf{u}^{(0)}+\mathbf{u}^{(1)}+\cdots \mathbf{u}^{(n)}$ $+O\left(\epsilon^{(n+1)}\right)$, where each $\mathbf{u}^{(m)}$ is $O\left(\epsilon^{m}\right)$. Collecting terms at each order of $\epsilon$ in Eq. (6) gives the following recursion relation:

$$
u_{i}^{(n+1)}(\boldsymbol{x})=\int_{\Omega} G_{i j}\left(\boldsymbol{x}, \boldsymbol{x}^{\prime}\right) \partial_{k^{\prime}}\left[\delta c_{j k l m} \partial_{l^{\prime}} u_{m}^{(n)}\right]\left(\boldsymbol{x}^{\prime}\right) d^{D} \boldsymbol{x}^{\prime} .
$$

The boundary conditions used to define $\mathbf{G}$ guarantee that for all $n>0$, the displacements $\mathbf{u}^{(n)}$ are zero on the boundary $\partial \Omega$.

The quantity we are specifically seeking to establish is the elastic energy density $E^{\mathrm{el}}=\ell^{-D} \int_{\Omega} \frac{1}{2} \boldsymbol{\tau}(\boldsymbol{x}): \boldsymbol{\varepsilon}(\boldsymbol{x}) d^{D} \boldsymbol{x}$, where we recall that $\ell$ is the linear dimension of a mesovolume. The definitions of the strain $\varepsilon_{i j}=\frac{1}{2}\left(\partial_{i} u_{j}+\partial_{j} u_{i}\right)$ and stress $\tau_{i j}=\left(C_{i j k l}^{0}+\delta c_{i j k l}\right) \varepsilon_{k l}$ give immediately the following relations:

$$
\begin{gathered}
\varepsilon_{i j}^{(n)}=\frac{1}{2}\left(\partial_{i} u_{j}^{(n)}+\partial_{j} u_{i}^{(n)}\right), \\
\tau_{i j}^{(n)}=C_{i j k l}^{0} \varepsilon_{k l}^{(n)}+\delta c_{i j k l} \varepsilon_{k l}^{(n-1)},
\end{gathered}
$$

$$
E^{(n)}=\frac{1}{2 \ell^{D}} \sum_{a=0}^{n} \int_{\Omega} \boldsymbol{\tau}^{(n-a)}: \boldsymbol{\varepsilon}^{(a)} d^{D} \boldsymbol{x}
$$

with the convention $\boldsymbol{\varepsilon}^{(-1)}=0$. In the last expression, the fact that $\mathbf{u}^{(a)}=0$ on the boundary for all $a>0$ guarantees that after integrating by parts,

$$
\begin{aligned}
\int_{\Omega} \boldsymbol{\tau}^{(n-a)}: \boldsymbol{\varepsilon}^{(a)} d^{D} \boldsymbol{x} & =\int_{\Omega} \boldsymbol{\tau}^{(n-a)}: \nabla \mathbf{u}^{(a)} d^{D} \boldsymbol{x} \\
& =\int_{\partial \Omega} \mathbf{n} \cdot \boldsymbol{\tau}^{(n-a)} \cdot \mathbf{u}^{(a)} d^{D-1} \boldsymbol{x}=0,
\end{aligned}
$$

where we used the facts that the stress tensor is symmetric and solenoidal. The $n$th term of the total elastic energy is then

$$
E^{(n)}=\frac{1}{2} \overline{\tau^{(n)}}: \boldsymbol{\varepsilon}^{(0)}
$$

where the upper bar denotes a volume average over $\Omega$.

The first term of the elastic energy is independent of the $\varphi$ field, and corresponds to the physically unimportant amount of energy

$$
E^{(0)}=\frac{1}{2} \boldsymbol{\varepsilon}^{(0)}: \mathbf{C}^{(0)}: \boldsymbol{\varepsilon}^{(0)}
$$

stored in the intact state.

For the higher orders $n \geqslant 1, \tau^{(n)}$ is expressed by Eq. (9), and the same argument as above using the fact that $\mathbf{u}^{(n)}=0$ on $\partial \Omega$ eliminates a term:

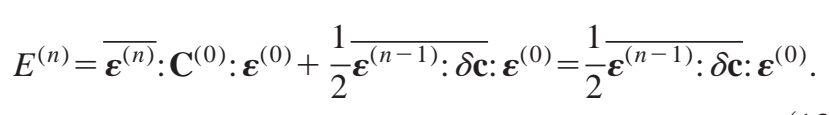

The second term of the developement,

$$
E^{(1)}=\frac{1}{2} \boldsymbol{\varepsilon}^{(0)}: \overline{\delta \mathbf{c}}: \boldsymbol{\varepsilon}^{(0)}
$$

represents only a local dependance on $\delta \mathbf{c}$ (and therefore on the crack field) since it does not involve nested integrals over two different positions. It will be shown to represent only the contribution of the average crack porosity to the stiffness of the rock.

The third term of the development is where the desired crack-to-crack interactions arrive. Using the symmetry of $\delta \mathbf{c}$ under the inversion of its two first or last indices, and Eq. (7) to have an integral form of $\mathbf{u}^{(1)}$, Eq. (13) transforms to

$$
\begin{aligned}
2 \ell^{D} E^{(2)}= & \int \partial_{b} u_{a}^{(1)}(\boldsymbol{x}) \delta c_{a b c d}(\boldsymbol{x}) \varepsilon_{c d}^{(0)} d^{D} \boldsymbol{x} \\
= & \iint \varepsilon_{c d}^{(0)} \delta c_{a b c d}(\boldsymbol{x}) \partial_{b} G_{a j}\left(\boldsymbol{x}, \boldsymbol{x}^{\prime}\right) \\
& \times \partial_{k^{\prime}} \delta c_{j k l m}\left(\boldsymbol{x}^{\prime}\right) \varepsilon_{l m}^{(0)} d^{D} \boldsymbol{x} d^{D} \boldsymbol{x}^{\prime}
\end{aligned}
$$


This term accounts for the way that a crack present at $\boldsymbol{x}^{\prime}$ energetically interacts with a different crack at $\boldsymbol{x}$. This is the nonlocal interaction term that is ultimately responsible for the localization transition. The higher terms of the Born development can be neglected for our purposes.

\section{B. Elastic energy as explicit function of the crack field}

To establish the terms of the Born-approximated elastic energy as explicit functions of both the crack state $\varphi$ and the imposed strain $\boldsymbol{\varepsilon}^{(0)}$, a few definitions are first introduced.

The principal axes of $\boldsymbol{\varepsilon}^{(0)}$ are along $\left(\hat{\mathbf{e}}_{\text {radial }}, \hat{\mathbf{e}}_{\text {axial }}\right)$ as denoted in Fig. 1. Our square network of cells is rotated $+45^{\circ}$ from this orthonormal basis. We work here in the coordinates $\left(\hat{\mathbf{e}}_{1}, \hat{\mathbf{e}}_{2}\right)$ of the square network so that the applied-strain takes the form

$$
\boldsymbol{\varepsilon}^{(0)}=\frac{1}{2}\left(\begin{array}{cc}
\Delta & \gamma \\
\gamma & \Delta
\end{array}\right)
$$

where $\Delta=\varepsilon_{\text {radial }}+\varepsilon_{\text {axial }}$ and $\gamma=-\left(\varepsilon_{\text {radial }}-\varepsilon_{\text {axial }}\right)$ are the imposed dilatation and shear strain.

For convenience, we assume the intact material to be isotropic. Taking $\lambda+2 \mu$ as the stress unit, where $(\lambda, \mu)$ are the Lamé parameters of the material, and using the usual tensorto-matrix mapping of the indices (11) $\rightarrow 1$; (22) $\rightarrow 2$; (12) $\rightarrow 3$, the fourth-order stiffness tensor of the intact material takes the form

$$
\mathbf{C}^{0}=\left(\begin{array}{ccc}
1 & 2 \alpha-1 & 0 \\
2 \alpha-1 & 1 & 0 \\
0 & 0 & 1-\alpha
\end{array}\right),
$$

where

$$
\alpha=\frac{\lambda+\mu}{\lambda+2 \mu}
$$

is a material-dependent constant in the range $[0.5,1]$.

The deviation $\delta \mathbf{c}$ of this tensor due to the possible presence of a crack in a cell separates into an isotropic contribution independent of the crack's orientation, and into an anisotropic orientation-dependent contribution. In the Appendix, we demonstrate that

$$
\begin{aligned}
& \delta \mathbf{c}(\boldsymbol{x})=\epsilon[\mathbf{A} \varphi(\boldsymbol{x})+\mathbf{B}|\varphi(\boldsymbol{x})|], \\
& \mathbf{A}=\left(\begin{array}{ccc}
\eta_{2}-\eta_{1} & 0 & 0 \\
0 & \eta_{1}-\eta_{2} & 0 \\
0 & 0 & 0
\end{array}\right), \\
& \mathbf{B}=\left(\begin{array}{ccc}
-\eta_{1} & -(2 \alpha-1) \eta_{2} & 0 \\
-(2 \alpha-1) \eta_{2} & -\eta_{1} & 0 \\
0 & 0 & -(1-\alpha) \eta_{3}
\end{array}\right) \text {, }
\end{aligned}
$$

where $\left(\eta_{1}, \eta_{2}, \eta_{3}\right)$ are positive constants expressed in the Appendix in terms of the Lamé parameters.
Making the necessary contractions over the indices, we easily obtain the trivial (crack independent) energy $E^{(0)}$ using Eqs. (12), (16), and (17). For later convenience, this result is best written in matrix form as

$$
\begin{gathered}
E^{(0)}=\frac{1}{2}(\Delta, \gamma) \cdot \mathbf{M}_{0} \cdot(\Delta, \gamma)^{T}, \\
\mathbf{M}_{0}=\left(\begin{array}{cc}
\alpha & 0 \\
0 & 1-\alpha
\end{array}\right) .
\end{gathered}
$$

Using the auxiliary field

$$
\psi(x)=|\varphi(x)|
$$

denoting the amplitude of each crack, one similarly obtains [using Eqs. (14) and (19)-(21)]

$$
\begin{aligned}
E^{(1)} & =\frac{\epsilon}{2}\left[\boldsymbol{\varepsilon}^{(0)}: \mathbf{A}: \boldsymbol{\varepsilon}^{(0)} \bar{\varphi}+\boldsymbol{\varepsilon}^{(0)}: \mathbf{B}: \boldsymbol{\varepsilon}^{(0)} \bar{\psi}\right] \\
& =\frac{1}{2}(\Delta, \gamma) \cdot \mathbf{M}_{1} \cdot(\Delta, \gamma)^{T},
\end{aligned}
$$

with

$$
\begin{gathered}
\mathbf{M}_{1}=-\epsilon \bar{\psi}\left(\begin{array}{cc}
\kappa_{2} & 0 \\
0 & \kappa_{3}
\end{array}\right), \\
\kappa_{2}=\frac{\eta_{1}}{2}+\frac{2 \alpha-1}{2} \eta_{2}, \\
\kappa_{3}=(1-\alpha) \eta_{3} .
\end{gathered}
$$

The term proportional to $\bar{\varphi}$ has algebraically canceled due to the symmetry of the problem under parity; inversion of the minor axis $\hat{\mathbf{e}}_{\text {radial }}$ flips the orientation of cracks, and therefore changes the sign of $\bar{\varphi}$, while the energy remains necessarily unchanged. The surviving term is negative and proportional to $\bar{\psi}$, and accounts for the softening of the mesovolume due to the presence of cracks. This dependence on the total number of cracks is the only order-parameter dependent effect to first order.

Last, the crack-interaction term of principal interest can be readily expressed from Eqs. (15) and (19) as

$$
\begin{aligned}
-2 \ell^{D} E^{(2)}= & \epsilon^{2} \varepsilon_{c d}^{(0)} \varepsilon_{k l}^{(0)} A_{a b c d} A_{i j k l} f_{a i b j} \\
& +2 \epsilon^{2} \varepsilon_{c d}^{(0)} \varepsilon_{k l}^{(0)} A_{a b c d} B_{i j k l} g_{a i b j} \\
& +\epsilon^{2} \varepsilon_{c d}^{(0)} \varepsilon_{k l}^{(0)} A_{a b c d} B_{i j k l} h_{a i b j},
\end{aligned}
$$

where the fourth-order tensors $\mathbf{f}, \mathbf{g}, \mathbf{h}$ are functionals of $\varphi$ and defined as

$$
\begin{aligned}
& f_{a i b j}=\int d^{D} \boldsymbol{x} \int d^{D} \boldsymbol{x}^{\prime} G_{a i}\left(\boldsymbol{x}, \boldsymbol{x}^{\prime}\right) \partial_{b} \varphi \partial_{j^{\prime}} \varphi, \\
& g_{a i b j}=\int d^{D} \boldsymbol{x} \int d^{D} \boldsymbol{x}^{\prime} G_{\{a i\}}\left(\boldsymbol{x}, \boldsymbol{x}^{\prime}\right) \partial_{b} \varphi \partial_{j^{\prime}} \psi,
\end{aligned}
$$




$$
h_{a i b j}=\int d^{D} \boldsymbol{x} \int d^{D} \boldsymbol{x}^{\prime} G_{a i}\left(\boldsymbol{x}, \boldsymbol{x}^{\prime}\right) \partial_{b} \psi \partial_{j^{\prime}} \psi
$$

In the second term, the reciprocity of the Green function $G_{a i}\left(\boldsymbol{x}, \boldsymbol{x}^{\prime}\right)=G_{a i}\left(\boldsymbol{x}^{\prime}, \boldsymbol{x}\right)$ is used as well as the notation $G_{\{a i\}}$ $=\left(G_{a i}+G_{i a}\right) / 2$.

The Green tensor needed here satisfies the Dirichlet conditions of Eq. (5) and can be obtained, in principle, from the infinite-space Green tensor via the image method. However, this transforms $E^{(2)}$ into an infinite series (one term for each image), and makes the functional integrations of Paper III analytically hopeless. To remedy this problem, the Green function with a periodic instead of zero boundary condition is used as ersatz. Since $\mathbf{u}^{(1)}$ is only affected close to the boundaries by this replacement, this approximation will be considered valid for the evaluation of the volume integral $E^{(2)}$.

The double integrals of Eqs. (30)-(32) are most easily expressed using the $2 \mathrm{D}$ finite-Fourier transform

$$
\begin{aligned}
& \tilde{F}(\boldsymbol{k})=\int_{\Omega} d^{D} \boldsymbol{x} F(\boldsymbol{x}) e^{-i \boldsymbol{k} \cdot \boldsymbol{x}}, \\
& F(\boldsymbol{x})=\frac{1}{\ell^{D}} \sum_{\boldsymbol{k}} \tilde{F}(\boldsymbol{k}) e^{i \boldsymbol{k} \cdot \boldsymbol{x}},
\end{aligned}
$$

where the sum over the wave vectors $\boldsymbol{k}$ is over $\{\boldsymbol{k}$ $\left.=2 \pi n_{i} / \ell \hat{\mathbf{e}}_{i} ; \forall i, n_{i} \in \mathbb{Z}\right\}$ with an upper cutoff given by $\max n_{i}>\ell / \Lambda$ that reflects the fact that the order parameter cannot vary on scales smaller than cell sizes $\Lambda$. Since the Green function used is defined with periodic boundary conditions, it satisfies $G_{a i}\left(\boldsymbol{x}, \boldsymbol{x}^{\prime}\right)=G_{a i}\left(\boldsymbol{x}-\boldsymbol{x}^{\prime}\right)$. Its Fourier transform is easily established, and upon recalling that $(\lambda+2 \mu)$ is adopted as the stress unit, reads

$$
\begin{gathered}
\widetilde{\mathbf{G}}(\boldsymbol{k})=\frac{1}{(1-\alpha) \boldsymbol{k}^{2}}(\mathbf{I}-\alpha \hat{\boldsymbol{k}} \hat{\boldsymbol{k}}), \\
\hat{\boldsymbol{k}}=\frac{\boldsymbol{k}}{\|\boldsymbol{k}\|},
\end{gathered}
$$

where $\mathbf{I}$ is the identity tensor. This is real and symmetric, as is $\mathbf{G}(\boldsymbol{x})$ itself. Since $\varphi$ and $\psi$ are real fields, one has $\tilde{\varphi}$ $(-\boldsymbol{k})=\tilde{\varphi}^{*}(\boldsymbol{k})$ and $\widetilde{\psi}(-\boldsymbol{k})=\widetilde{\psi}^{*}(\boldsymbol{k})$. Using these relations, together with the identity $\int_{\Omega} d^{D} \boldsymbol{x} e^{i k \cdot x}=\ell^{D} \delta_{k}$, the integrals of Eqs. (30)-(32) become the following sums

$$
\begin{gathered}
f_{a i b j}=\frac{1}{\ell^{D}} \sum_{\boldsymbol{k} \neq 0}|\tilde{\varphi}(\boldsymbol{k})|^{2} \hat{k}_{j} \hat{k}_{b}\left(\delta_{i a}-\alpha \hat{k}_{i} \hat{k}_{a}\right) \\
g_{a i b j}=\frac{1}{\ell^{D}} \sum_{\boldsymbol{k} \neq 0} \operatorname{Re}\left[\tilde{\varphi}(\boldsymbol{k}) \tilde{\psi}^{*}(\boldsymbol{k})\right] \hat{k}_{j} \hat{k}_{b}\left(\delta_{i a}-\alpha \hat{k}_{i} \hat{k}_{a}\right), \\
h_{a i b j}=\frac{1}{\ell^{D}} \sum_{\boldsymbol{k} \neq 0}|\tilde{\psi}(\boldsymbol{k})|^{2} \hat{k}_{j} \hat{k}_{b}\left(\delta_{i a}-\alpha \hat{k}_{i} \hat{k}_{a}\right),
\end{gathered}
$$

where Re denotes the real part of a complex quantity and where $\hat{k}_{i}$ denotes the $i$ th component of $\hat{\mathbf{k}}=\boldsymbol{k} /\|\boldsymbol{k}\|$. With the following definitions associated with the orientation of $\boldsymbol{k}$ :

$$
\begin{gathered}
\theta_{\boldsymbol{k}}=\left(\hat{\boldsymbol{e}}_{1}, \boldsymbol{k}\right), \\
u_{\boldsymbol{k}}=\cos \left(2 \theta_{\boldsymbol{k}}\right)=\cos ^{2} \theta_{\boldsymbol{k}}-\sin ^{2} \theta_{\boldsymbol{k}}=\hat{k}_{1}^{2}-\hat{k}_{2}^{2}, \\
v_{\boldsymbol{k}}=\sin \left(2 \theta_{\boldsymbol{k}}\right)=2 \cos \theta_{\boldsymbol{k}} \sin \theta_{\boldsymbol{k}}=2 \hat{k}_{1} \hat{k}_{2},
\end{gathered}
$$

the remaining contraction in Eq. (29) over the eight indices (abcdijkl) is performed. The calculation is a bit long but without surprise and finally produces

$$
\begin{aligned}
E^{(2)} & =\frac{1}{2}(\Delta, \gamma) \cdot \mathbf{M}_{2} \cdot(\Delta, \gamma)^{T}, \\
\mathbf{M}_{2} & =\frac{-\epsilon^{2}}{(1-\alpha) \ell^{2 D}}\left(\begin{array}{ll}
a & b \\
b & c
\end{array}\right),
\end{aligned}
$$

where the components $a, b$, and $c$ are defined,

$$
\begin{gathered}
a=\sum_{k \neq 0} a_{k} ; \quad b=\sum_{k \neq 0} b_{k} ; \quad c=\sum_{k \neq 0} c_{k}, \\
a_{k}=\left(1-\alpha u_{k}^{2}\right) \kappa_{1}^{2}\left|\tilde{\varphi}_{k}\right|^{2}+2(1-\alpha) u_{k} \kappa_{1} \kappa_{2} \operatorname{Re}\left(\widetilde{\varphi}_{k} \widetilde{\psi}_{k}^{*}\right) \\
+(1-\alpha) \kappa_{2}^{2}\left|\widetilde{\psi}_{k}\right|^{2}, \\
b_{k}=-\alpha u_{k} v_{k} \kappa_{1} \kappa_{3} \operatorname{Re}\left(\tilde{\varphi}_{k} \widetilde{\psi}_{k}^{*}\right)+(1-\alpha) v_{k} \kappa_{2} \kappa_{3}\left|\widetilde{\psi}_{k}\right|^{2}, \\
c_{k}=\left(1-\alpha v_{k}^{2}\right) \kappa_{3}^{2}\left|\widetilde{\psi}_{k}\right|^{2},
\end{gathered}
$$

with $\kappa_{2}, \kappa_{3}$ defined in Eqs. (27), (28) and $\kappa_{1}$ a new materialdependent constant,

$$
\kappa_{1} \hat{=} \frac{\eta_{1}-\eta_{2}}{2}
$$

\section{SURFACE FORMATION ENERGY}

Next, we must account for the energy $E_{j}^{I}$ that irreversibly went into creating the cracks of a given crack state $j$ at a maximum deformation $\boldsymbol{\varepsilon}_{m}$. In Paper I, this contribution was obtained using Griffith's criterion as

$$
E_{j}^{I}=\frac{q}{2} \ell^{D} \boldsymbol{\varepsilon}_{m}:\left(\mathbf{C}_{0}-\mathbf{C}_{j}\right): \boldsymbol{\varepsilon}_{m}
$$

where $q$ derives from the quenched disorder and is bounded as $0.5 \leqslant q<1$. The derivation of this statement implicitly assumed that all cracks were the same length. In the present treatment, cracks are allowed to have any length in the range $0 \leqslant d \leqslant d_{m}$. It is a straightforward exercise to demonstrate that if the breaking energies for each possible length $d$ are all sampled from the same quenched-disorder distribution, then Eq. (47) again holds. We forego such a demonstration. In the notation of the present paper we may thus state that 


$$
E[\varphi]^{I}=q\left(E^{(0)}-E^{\mathrm{el}}[\varphi]\right)=-q\left(E^{(1)}+E^{(2)}\right)[\varphi],
$$

where $E^{(1)}$ and $E^{(2)}$ are the terms of the Born-development given by Eqs. (25) and (43) upon replacing the current strain parameters $\Delta$ and $\gamma$ by the maximum-achieved strain $\Delta_{m}$ and $\gamma_{m}$.

\section{TEMPERATURE}

Although not required as part of the Hamiltonian model, we now give an explicit in $\Delta_{m}, \gamma_{m}$ approximate expression for the temperature by using Eq. (59) of Paper I. This temperature was derived in Paper I assuming only a single crack size. Unfortunately, the result does not easily generalize to multiple crack sizes and so we simply take $d=d_{m}$ to obtain the estimate

$$
\ell^{D} T\left(\Delta_{m}, \gamma_{m}\right)=-\frac{(1-q) d_{m}^{D} e_{1}\left(\Delta_{m}, \gamma_{m}\right)}{\ln \left\{\left[\zeta / e_{1}\left(\Delta_{m}, \gamma_{m}\right)\right]^{q /(1-q)}-1\right\}},
$$

where $e_{1} d_{m}^{D}$ is how much the first-Born elastic energy in a mesovolume is reduced when a crack of length $d_{m}$ is introduced [c.f. Eqs. (25) and (26)]. The energy density $e_{1}\left(\Delta_{m}, \gamma_{m}\right)$ is defined as

$$
e_{1}\left(\Delta_{m}, \gamma_{m}\right)=\frac{1}{2}\left(\kappa_{2} \Delta_{m}^{2}+\kappa_{3} \gamma_{m}^{2}\right)
$$

while $\zeta$ is a dimensionless "fracture toughness" parameter defined as

$$
\zeta \equiv \frac{\Gamma}{(\lambda+2 \mu) d_{m}} .
$$

There is a phase transition when $\left(\zeta / e_{1}\right)^{q /(1-q)}=2$ and $T$ diverges so that all crack states become equally probable.

We now consider whether such a phase transition is expected in laboratory experiments on rocks. The order of magnitude values $\Gamma \sim 10^{2} \mathrm{~J} / \mathrm{m}^{2}, d_{m} \sim 10^{-5} \mathrm{~m}, \kappa_{2} \sim 1$, and $(\lambda$ $+2 \mu) \sim 10^{10} \mathrm{~Pa}$ are appropriate for typical grains in rocks so that $\zeta \sim 10^{-3}$. When a rock fails in shear, the accumulated strain is on the order of a percent or two, so that the maximum value of $e_{1}$ of interest is also on the order of $10^{-4}$. We thus find that at shear failure, $\zeta / e_{1} \sim 10$ and so we a priori expect the localization transition to occur prior to the temperature-divergence transition. This is more quantitatively demonstrated in Paper III.

\section{SUMMARY}

Collecting together both the elastic energy and the surface formation energy, we obtain at last the Hamiltonian to be used in performing ensemble averages over crack states in the next paper. We write this Hamiltonian in the final form

$$
\begin{gathered}
E_{j}\left(\boldsymbol{\varepsilon}, \boldsymbol{\varepsilon}_{m}\right)=E^{R}(\boldsymbol{\varepsilon})[\varphi]+E^{I}\left(\boldsymbol{\varepsilon}_{m}\right)[\varphi], \\
E^{R}(\boldsymbol{\varepsilon})[\varphi]=E^{0}(\Delta, \gamma)+E^{\mathrm{av}}(\Delta, \gamma)[\varphi]+E^{\mathrm{int}}(\Delta, \gamma)[\varphi], \\
E^{I}\left(\boldsymbol{\varepsilon}_{m}\right)[\varphi]=-q\left\{E^{\mathrm{av}}\left(\Delta_{m}, \gamma_{m}\right)[\varphi]+E^{\mathrm{int}}\left(\Delta_{m}, \gamma_{m}\right)[\varphi]\right\},
\end{gathered}
$$

where $(\Delta, \gamma)$ are the isotropic and shear strain components of the current strain tensor $\boldsymbol{\varepsilon}$, and $\left(\Delta_{m}, \gamma_{m}\right)$ are similar quantities referring to the maximum achieved strain $\boldsymbol{\varepsilon}_{m}$. The en$\operatorname{ergy} E^{0}$ is the trivial elastic energy of the uncracked state

$$
E^{0}(\Delta, \gamma)=\frac{1}{2}\left\{\alpha \Delta^{2}+(1-\alpha) \gamma^{2}\right\}
$$

where $\alpha$ is a dimensionless elastic constant in the range $[0.5,1]$ defined by Eq. (18).

The next term in the Born development is $E^{\mathrm{av}}=E^{(1)}$, which depends only on the volume average $\bar{\psi}$, which is the fraction of cracked cells in the crack state $\varphi$ and is thus entirely independent of the spatial fluctuations of $\varphi$. Its dependence on the strain $(\Delta, \gamma)$ is

$$
E^{\mathrm{av}}(\Delta, \gamma)[\varphi]=-\frac{1}{2}\left[\kappa_{2} \Delta^{2}+\kappa_{3} \gamma^{2}\right] \epsilon \bar{\psi}
$$

We defined $\epsilon=\left(d_{m} / \Lambda\right)^{D}$ to be a small parameter, where $D$ $=2$ is the number of space dimensions in the model, and $d_{m}, \Lambda$, and $\ell$ as respectively the linear sizes of the largest crack, a unit cell, and a mesovolume. The three coefficients $\kappa_{i}$ are positive dimensionless material-dependent constants defined by Eqs. (27), (28), and (46).

The interaction energy $E^{\text {int }}=E^{(2)}$ involve a quadratic matrix operator $P_{\boldsymbol{k}}$ that, for each nonzero wave vector $\boldsymbol{k}$, mixes together the Fourier modes of both $\varphi$ and $\psi$ :

$$
\begin{gathered}
E^{\mathrm{int}}(\Delta, \gamma)[\varphi]=\frac{-\epsilon^{2}}{2(1-\alpha) \ell^{2 D}} \sum_{k \neq 0}\left(R_{k}^{T} \cdot P_{k} \cdot R_{k}+I_{k}^{T} \cdot P_{k} \cdot I_{k}\right), \\
R_{k}=\left[\operatorname{Re}\left(\tilde{\varphi}_{k}\right) ; \operatorname{Re}\left(\widetilde{\psi}_{k}\right)\right]^{T}, \\
I_{k}=\left[\operatorname{Im}\left(\tilde{\varphi}_{k}\right) ; \operatorname{Im}\left(\widetilde{\psi}_{k}\right)\right]^{T}, \\
P_{k}=\left[\begin{array}{cc}
L_{k} & M_{k} \\
M_{k} & N_{k}
\end{array}\right],
\end{gathered}
$$

where $\mathrm{Re}$ and Im represent the real and imaginary part of a complex number. The components $L_{k}, M_{k}, N_{k}$ depend both on the applied-strain parameters (maximum or actual ones), and the wave vector $\boldsymbol{k}$. In anticipation of Paper III, it is convenient to introduce

$$
\omega=\frac{\kappa_{3}}{\kappa_{1}} \frac{\gamma}{\Delta}
$$

as the shear-strain variable and to define the parameter

$$
c=\kappa_{2} / \kappa_{1}=1+\frac{2 \mu(\lambda+\mu)\left(\lambda_{+} 2 \mu\right)}{\lambda^{3}},
$$


where $c>1$. The components of the matrix $P_{\boldsymbol{k}}$ are then

$$
\begin{gathered}
L_{k}(\Delta, \omega)=\Delta^{2} \kappa_{1}^{2}\left(1-\alpha u_{k}^{2}\right), \\
M_{k}(\Delta, \omega)=\Delta^{2} \kappa_{1}^{2} u_{k}\left[(1-\alpha) c-\alpha v_{k} \omega\right], \\
N_{k}(\Delta, \omega)=\Delta^{2} \kappa_{1}^{2}\left[(1-\alpha) c^{2}+2(1-\alpha) c v_{k} \omega\right. \\
\left.+\left(1-\alpha v_{k}^{2}\right) \omega^{2}\right],
\end{gathered}
$$

where $u_{k}=\cos \left(2 \theta_{k}\right)$ and $v_{k}=\sin \left(2 \theta_{k}\right)$ are functions that characterize the orientation of $\boldsymbol{k}$ through its polar angle $\theta_{\boldsymbol{k}}$.

Note that all terms contributing to the Hamiltonian have been written in a dimensionless form in which energy density $E_{j}$, like stress, is measured in units of $(\lambda+2 \mu)$.

\section{APPENDIX: EFFECTIVE MODULI OF A CRACKED CELL}

A crack is modeled here as an elongated ellipse having a major axis of length $d$ and a minor axes of length $w$ in the limit that $w / d \ll 1$ which corresponds to a so-called "pennyshaped" crack. Its long axis is by convention oriented along $\hat{\mathbf{e}}_{1}$ if locally $\varphi>0$, and along $\hat{\mathbf{e}}_{2}$ if $\varphi<0$. The unit cell is a square whose sides are colinear with $\left(\hat{\mathbf{e}}_{1} ; \hat{\mathbf{e}}_{2}\right)$, and has a size $\Lambda \gg d$ since the crack is taken to be small. The interior of the crack is supposed to be much more compliant than the embedding matrix and all plastic deformation will be ignored; i.e., there is no residual stress or strain allowed for in the cracked system when it is unloaded to zero applied stress.

Denoting as usual the volume average of a quantity with an overbar, we seek to determine the elastic-stiffness tensor $\mathbf{C}$ of a cell as defined through the relation

$$
\bar{\tau}_{i j}=C_{i j k l} \bar{\varepsilon}_{k l}
$$

The region inside the crack is occupied by a uniform material of stiffness $\mathbf{C}^{1}$ while the intact matrix surrounding the crack is occupied by a material of stiffness $\mathbf{C}^{1}$. Upon denoting $v$ the volume fraction of the crack in the cell, we obtain directly

$$
\bar{\tau}_{i j}=(1-v) C_{i j k l}^{0} \bar{\varepsilon}_{k l}^{0}+v C_{i j k l}^{1} \bar{\varepsilon}_{k l}^{1} .
$$

Eshelby [11] demonstrates that the strain $\boldsymbol{\varepsilon}^{1}$ inside an elliptic inclusion is uniform while $\mathrm{Wu}$ [12] relates this strain to the strain at infinity by a tensor $\mathbf{T}$,

$$
\bar{\varepsilon}_{i j}^{1}=T_{i j k l} \varepsilon_{k l}^{\infty} \text {. }
$$

With cracks considered as small inclusions in their embedding cell $(v \ll 1)$, the approximation $\boldsymbol{\varepsilon}^{\infty} \simeq \overline{\boldsymbol{\varepsilon}}$ is valid to leading order in the above, so that

$$
\begin{gathered}
\bar{\varepsilon}_{i j}^{0}=W_{i j k l} \bar{\varepsilon}_{k l}, \\
(1-v) W_{i j k l}=\left(\delta_{i k} \delta_{j l}-v T_{i j k l}\right) .
\end{gathered}
$$

Using Eqs. (A3) and (A4) for the average deformation in and out of the inclusion, Eq. (A2) has the desired linear form of Eq. (A1) with an effective stiffness tensor given by

$$
C_{i j k l}=C_{i j k l}^{0}-v\left(C_{i j m n}^{0}-C_{i j m n}^{1}\right) T_{m n k l} \simeq C_{i j k l}^{0}-v C_{i j m n}^{0} T_{m n k l} .
$$

This approximation is justified under the hypothesis that the material inside the inclusion (air) is far more compliant than the host material (solid silicate). These relations are valid in any space dimension $D$. The two-dimensional case of interest to us here can be obtained from the three-dimensional Wu-Eshelby results by working with a three-dimensional ellipsoidal inclusion having semiaxes of linear dimension $a$ $=d / 2 ; b=w / 2$; and $c=h / 2$ embedded within a cell of dimension $\Lambda \times \Lambda \times h$ in the limit that $h \gg \Lambda$. In this limit, the three-dimensional problem becomes one in two dimensions.

$\mathrm{Wu}$ expresses his tensor $\mathbf{T}$ in terms of a tensor $\mathbf{S}$ defined by Eshelby,

$$
\begin{gathered}
T_{i j i j}=\frac{1}{2\left(1-2 S_{i j i j}\right)} \quad \text { when } i \neq j, \\
\left(\begin{array}{lll}
T_{1111} & T_{1122} & T_{1133} \\
T_{2211} & T_{2222} & T_{2233} \\
T_{3311} & T_{3322} & T_{3333}
\end{array}\right) \\
=\left(\begin{array}{ccc}
1-S_{1111} & -S_{1122} & -S_{1133} \\
-S_{2211} & 1-S_{2222} & -S_{2233} \\
-S_{3311} & -S_{3322} & 1-S_{3333}
\end{array}\right)^{-1}
\end{gathered}
$$

The Eshelby [11] tensor components are defined

$$
\begin{gathered}
S_{1111}=Q a^{2} I_{a a}+R I_{a}, \\
S_{1122}=Q b^{2} I_{a b}-R I_{a}, \\
S_{1212}=\frac{Q}{2}\left(a^{2}+b^{2}\right) I_{a b}+\frac{R}{2}\left(I_{a}+I_{b}\right),
\end{gathered}
$$

with similar expressions for the remaining components obtained through the permutation of $a, b, c$ and 1,2,3. In the notation of the present paper, the various parameters of Eqs. (A9)-(A11) are defined

$$
Q=\frac{3}{8 \pi\left(1-\sigma_{p}\right)} \text { and } R=\frac{1-2 \sigma_{p}}{8 \pi\left(1-\sigma_{p}\right)},
$$

where $\sigma_{p}=\lambda / 2(\lambda+\mu)$ is the Poisson's ratio of the solid material (assumed isotropic), and

$$
\begin{gathered}
I_{a}=2 \pi a b \int_{0}^{\infty} \frac{d u}{\left(a^{2}+u\right) D}, \\
I_{a a}=2 \pi a b \int_{0}^{\infty} \frac{d u}{\left(a^{2}+u\right)^{2} D},
\end{gathered}
$$




$$
I_{a b}=\frac{2}{3} \pi a b \int_{0}^{\infty} \frac{d u}{\left(a^{2}+u\right)\left(b^{2}+u\right) D}
$$

with $D=\sqrt{\left(a^{2}+u\right)\left(b^{2}+u\right)}$ and $I_{c}=I_{a c}=I_{b c}=I_{c c}=0$. Similar expressions are obtained for $I_{b}$ and $I_{b b}$ by replacing $a$ and $b$ in the above. These elliptic integrals are evaluated to the leading order in the small aspect ratio $\delta=b / a$ which gives

$$
\begin{gathered}
I_{a}=4 \pi \delta, \quad I_{b}=4 \pi(1-\delta), \\
I_{a a}=\frac{4 \pi}{3 a^{2}} 2 \delta, \quad I_{b b}=\frac{4 \pi}{3 b^{2}}, \quad I_{a b}=\frac{4 \pi}{3 a^{2}}(1-2 \delta) .
\end{gathered}
$$

Defining parameters $q$ and $r$ by

$$
q=4 \pi Q=\frac{3}{2\left(1-\sigma_{p}\right)} \quad \text { and } \quad r=4 \pi R=\frac{1-2 \sigma_{p}}{2\left(1-\sigma_{p}\right)},
$$

we obtain that to the leading order in $1 / \delta$,

$$
\begin{gathered}
T_{1212}=\frac{3}{4 q} \frac{1}{\delta}, \\
T_{2211}=\frac{\frac{q}{3}-r}{r\left(1+\frac{q}{3}-r\right)} \frac{1}{\delta}, \\
T_{2222}=\frac{1}{r\left(1+\frac{q}{3}-r\right)} \frac{1}{\delta} .
\end{gathered}
$$

All remaining components of $\mathbf{T}$ are either $O(1)$ and therefore negligible, or are unimportant for the components of $\mathbf{C}$ related to directions 1 and 2 .

To get finally the deviation $\delta \mathbf{c}$ of the effective elastic moduli of the cracked cell through Eq. (A6), we note first that

$$
v=\frac{4 \pi}{3} \frac{a b c}{\Lambda^{2} h}=\frac{2 \pi}{3} \frac{a^{2}}{\Lambda^{2}} \frac{b}{a}=\frac{\pi}{6} \frac{d^{2}}{\Lambda^{2}} \delta=\frac{\pi}{6} \epsilon \psi \delta
$$

where we recall that $d=2 a$ is the crack's length, $w=2 b$ its width, and $\delta$ its aspect ratio. It is through this expression that the small parameter $\epsilon=\left(d_{m} / \Lambda\right)^{2} \ll 1$ enters the Born series. Note that $\psi=|\varphi|=\left(d / d_{m}\right)^{2}$ characterizes the extent of the crack. The third dimension of $h=2 c$ goes to infinity in order to obtain the two-dimensional limit of this three-dimensional system.

Replacing $q$ and $r$ by their expressions in terms of the Lamé parameters $\lambda, \mu$, and using by convention $(\lambda+2 \mu)$ as the stress unit, the crack-induced perturbations of the cell moduli are

$$
\begin{gathered}
\delta c_{2222}=-v C_{2222}^{0} T_{2222}=-\frac{\pi}{6} \epsilon \psi \frac{\lambda+2 \mu}{\mu}, \\
\delta c_{1111}=-v C_{1122}^{0} T_{2211}=-\frac{\pi}{6} \epsilon \psi \frac{\lambda^{2}}{\mu(\lambda+2 \mu)}, \\
\delta c_{1122}=-v C_{1122}^{0} T_{2222}=-\frac{\pi}{6} \epsilon \psi \frac{\lambda}{\mu}, \\
\delta c_{2211}=-v C_{2222}^{0} T_{2211}=-\frac{\pi}{6} \epsilon \psi \frac{\lambda}{\mu},
\end{gathered}
$$

$$
\delta c_{1212}=-v\left(C_{1212}^{0} T_{1212}+C_{1221}^{0} T_{2112}\right)=-\frac{\pi}{6} \epsilon \psi \frac{1}{2} \frac{\mu}{\lambda+\mu},
$$

with all other terms being zero except those obtained by the necessary symmetries under exchange of the two first or two last indexes.

Using the dimensionless constants $\alpha$ defined in Eq. (18) and introducing the positive dimensionless coefficients $\eta_{i}$,

$$
\begin{gathered}
\eta_{1}=\frac{\pi}{6} \frac{\lambda^{3}+\mu(\lambda+2 \mu)^{2}}{\lambda \mu(\lambda+2 \mu)}, \\
\eta_{2}=\frac{\pi}{6} \frac{\lambda+2 \mu}{\lambda}, \\
\eta_{3}=\frac{\pi}{12} \frac{\lambda+2 \mu}{\lambda+\mu}
\end{gathered}
$$

we obtain at last the deviation of the elastic moduli of a cell containing a crack with long axis oriented along $\hat{\mathbf{e}}_{1}$ (corresponding to a positive $\varphi$ ),

$$
\delta \mathbf{c}=\left(\begin{array}{ccc}
\eta_{2}-2 \eta_{1} & -(2 \alpha-1) \eta_{2} & 0 \\
-(2 \alpha-1) \eta_{2} & -\eta_{2} & 0 \\
0 & 0 & -(1-\alpha) \eta_{3}
\end{array}\right) \epsilon \psi .
$$

The expression for both possible orientations of the cracks is straightforward. Orienting the crack along $\hat{\mathbf{e}}_{2}$ instead $\hat{\mathbf{e}}_{1}$ is equivalent to exchanging the one and two indices in the components of $\delta \mathbf{c}$, which results in an exchange of the components $\delta c_{1111}$ and $\delta c_{2222}$, all remaining components of $\delta \mathbf{c}$ being unaffected by this change. Separating both expressions of $\delta \mathbf{c}$ into symmetric and antisymmetric parts, and noting that $\delta \mathbf{c}=0$ trivially when $\varphi=0$ (no crack), we obtain the general expression used in Eqs. (19)-(21). 
[1] P. Bésuelle, Ph.D. thesis, Université Joseph Fourier-Grenoble I, 1999, and references therein for a review.

[2] S. Arbabi and M. Sahimi, Phys. Rev. B 47, 695 (1993); M. Sahimi and S. Arbabi, ibid. 47, 703 (1993); 47, 713 (1993).

[3] G. G. Batrouni and A. Hansen, Phys. Rev. Lett. 80, 325 (1998).

[4] Statistical Models for the Fracture of Disordered Media, edited by H. J. Herrmann and S. Roux (North-Holland, Amsterdam, 1990).

[5] D. A. Lockner, in Rock Physics and Phase Relations, A Handbook of Physical Constants, edited by T. J. Ahrens (American Geophysical Union, Washington, DC, 1995), and references therein.
[6] R. L. Kranz, Tectonophysics 100, 449 (1983).

[7] M. L. Batzle, G. Simmons, and R. W. Siegfried, J. Geophys. Res. B 85, 7072 (1980).

[8] M. S. Paterson, Experimental Rock Deformation—the Brittle Field (Springer-Verlag, Berlin, 1978).

[9] D. A. Lockner, J. D. Byerlee, V. Kuksenko, A. Ponomarev, and A. Sidorin, in Fault Mechanics and Transport Properties of Rocks, edited by B. Evans and T. F. Wong (Academic Press, San Diego, 1992).

[10] K. Mair, I. Main, and S. Elphick, J. Struct. Geol. 22, 25 (2000).

[11] J. D. Eshelby, Proc. R. Soc. London, Ser. A 241, 376 (1957).

[12] T. T. Wu, Int. J. Solids Struct. 2, 1 (1966). 